\title{
Nuclear respiratory factor- $2 \alpha$ and adenosine triphosphate synapses in rat primary cortical neuron cultures: The key role of adenosine monophosphate-activated protein kinase
}

\author{
XIANG CHEN ${ }^{1}$, XIAO ZHAO ${ }^{2}$, MAONIAN ZHANG $^{1}$ and SHIHUI WEI ${ }^{1}$ \\ ${ }^{1}$ Department of Ophthalmology, The Chinese PLA General Hospital, Beijing 100853; \\ ${ }^{2}$ Department of Medical Affairs, Affiliated Hospital of The Chinese PLA General Hospital, Beijing 100048, P.R. China
}

Received October 11, 2014; Accepted June 26, 2015

DOI: $10.3892 / \mathrm{mmr} .2015 .4140$

\begin{abstract}
Nuclear respiratory factor- $2 \alpha(\mathrm{NRF}-2 \alpha)$ is an important transcription factor that regulates mitochondrial oxidative phosphorylation and regeneration. NRF-2 $\alpha$ regulates mitochondrial transcription factors (mTF)A and B, and mitochondrial DNA by indirectly regulating the mitochondrial respiratory enzyme chain subunit. In addition, NRF-2 $\alpha$ is involved in the mitochondrial energy metabolism. Peroxisome proliferator-activated receptor $\gamma$ coactivator $1 \alpha(\mathrm{PGC}-1 \alpha)$, is an important transcription coactivator of NRF-2 $\alpha$. Adenosine monophosphate-activated protein kinase (AMPK) is considered an important effector in the regulation of the energy metabolism balance of nervous system microenvironments. However, the signaling mechanism underlying the energy coupling of PGC- $1 \alpha$ and NRF- $2 \alpha$ in visual cortical neurons remains to be elucidated. The present study used a primary culture system of rat visual cortical neurons in order to investigate whether AMPK is involved in the regulation of NRF-2 $\alpha$ and PGC-1 $\alpha$ expression in cortical neurons. The results of the present study indicated that $\mathrm{KCl}$ depolarization rapidly activated AMPK, and significantly increased the expression levels of PGC-1 $\alpha$, NRF-2 $\alpha$ and mtTFA, as well as adenosine triphosphate production in cultured neurons. Similarly, the AMPK agonists 5-aminoimidazole-4-carboxamide riboside and resveratrol significantly increased the mRNA expression levels of PGC- $1 \alpha$ and NRF- $2 \alpha$ in cultured neurons. These responses were blocked by compound $\mathrm{C}$, an AMPK inhibitor. In conclusion, AMPK is an important transcriptional regulator of the neuronal excitation response, and exerts its regulatory effects via the PGC- $1 \alpha / \mathrm{NRF}-2 \alpha$ signaling pathway.
\end{abstract}

Correspondence to: Dr Xiao Zhao, Department of Medical Affairs, Affiliated Hospital of The Chinese PLA General Hospital, 51 Fucheng Road, Beijing 100048, P.R. China

E-mail:dr.zx@163.com

Key words: adenosine monophosphate-activated protein kinase, energy metabolism, mitochondria, neurons, nuclear respiratory factor- $2 \alpha$, visual cortex

\section{Introduction}

The brain is an organ with a high metabolic activity, and the normal functioning of neurons relies on a constant energy supply to the brain. Neurons are highly dependent on the mitochondrial oxidative system (OXPHOS) to produce adenosine triphosphate (ATP) (1). The structure, function and synapses of the neurons in the visual cortex change depending on environmental factors, a phenomenon know as experience-dependent plasticity. A previous study demonstrated that mitochondrial dysfunction may be an important factor in the inhibition of cortical neurons in visual disorders (2). In recent years, research on the mechanisms underlying visual disorders, such as amblyopia, and on the visual cortex have made significant progress. The mechanisms underlying the signal transduction pathways of the visual cortex are currently under investigation (3). Thus far, the signaling mechanisms underlying the neuronal activity responsible for energy coupling remains to be elucidated.

Nuclear respiratory factors (NRFs) are important transcription factors that regulate mitochondrial oxidative phosphorylation and regeneration (4). NRFs directly regulate mitochondrial respiration via the transcriptional regulation of respiratory enzyme chain subunit nuclear DNA (nDNA), and via the regulation of mitochondrial transcription factors (mTF)A and B, and mitochondrial DNA (mtDNA), thereby indirectly regulating the mitochondrial respiratory enzyme chain subunit (5). A previous study demonstrated that NRF-2 is involved in the mitochondrial energy coupling of visual cortical neurons (6). The mRNA and protein expression levels of NRF-2 were positively correlated with neuronal excitability, and concordant with cytochrome coxidase (COX) activity (6). In addition, peroxisome proliferator-activated receptor $\gamma$ coactivator $1 \alpha(\mathrm{PGC}-1 \alpha)$ is an important transcriptional coactivator, which is involved in the mitochondrial synthesis of ATP. A previous study demonstrated that PGC- $1 \alpha$ is able to induce NRF gene expression, thereby affecting OXPHOS expression (7). PGC- $1 \alpha$ is able to promote the transcription of NRFs genes, thereby changing mitochondrial respiratory chain function (8). However, the signaling mechanisms underlying the association between PGC-1 $\alpha$ and NRFs in the energy coupling process of visual cortical neurons remain to be investigated. 
Evidence from animal experiments and clinical observations indicates that adenosine monophosphate-activated protein kinase (AMPK) is an important effector in the regulation of the energy metabolism of nervous system microenvironments $(9,10)$. AMPK is an important regulator of mitochondrial and cell energy metabolism, and is associated with PGC-1 $\alpha$ (11). In addition, AMPK may be an important upstream signaling-activated molecule of the NRF transduction pathway. However, the role of AMPK in visual cortical neurons has yet to be elucidated. The present study hypothesized that the AMPK signaling pathway may have an important role in the energy coupling of visual cortical neurons.

The present study investigated the effects of neuronal activity on AMPK and its association with PGC- $1 \alpha$, NRF- $2 \alpha$, and mtTFA expression, as well as the role of mitochondria in a primary neuron culture system. The present study aimed to determine whether AMPK is involved in regulating the expression of NRF- $2 \alpha$ and PGC- $1 \alpha$ in primary visual cortical nerouns.

\section{Materials and methods}

Primary culture of visual cortical neurons. The present study was approved by the ethics committee of the Chinese PLA General Hospital (Beijing, China; no. 2012045). One day-old Sprague-Dawley rat pups (Experimental Animal Center, Chinese PLA General Hospital) were sacrificed by decapitation under $\mathrm{CO}_{2}$ anesthesia (10\% chloral hydrate; Sigma-Aldrich, St. Louis, MO, USA; $0.3 \mathrm{ml} / 100 \mathrm{~g}$; i.p.). The meninges were subsequently removed. The primary visual cortex (V1, area 17) was dissected under a Zeiss Axio Examiner microscope (Zeiss, Oberkochen, Germany). The tissue samples were then incubated for $10 \mathrm{~min}$ in trypsin/EDTA [0.05/0.02\%, $\mathrm{w} / \mathrm{v}$ in phosphate-buffered saline (PBS)] (Sigma-Aldrich) at $37^{\circ} \mathrm{C}$, and triturated in order to cause the dissociation of individual cells. The cells were plated onto $30-\mathrm{mm}$ plastic dishes coated with poly-L-lysine (Sigma-Aldrich) at a density of $5 \times 10^{5}$ cells $/ \mathrm{ml}$, in Dulbecco's modified Eagle's medium (Gibco Life Technologies, Carlsbad, CA, USA) supplemented with $10 \%$ fetal bovine serum (GE Healthcare Life Sciences, Logan, UT, USA) for $3 \mathrm{~h}$. The liquid medium was carefully discarded and replaced with a neurobasal medium (Gibco Life Technologies) supplemented with B27 solution (Gibco Life Technologies) and $0.5 \mathrm{mM} \mathrm{L}$-glutamine (Sigma-Aldrich), and cultured for $24 \mathrm{~h}$. A total of $2.5 \mu \mathrm{M}$ cytosine arabinoside (Sigma-Aldrich) was then added to the cultures to inhibit the replication of non-neuronal cells. The cultures were kept in cultivating medium at $37^{\circ} \mathrm{C}$ in an atmosphere containing $5 \% \mathrm{CO}_{2}$ for 5-6 days. The purity of the cultured neurons was $>95 \%$.

Membrane depolarization was conducted as previously described (12), and $25 \mathrm{mM} \mathrm{KCl}$ was added to the visual cortical neurons at days 5-6 of culture. PGC- $1 \alpha$, NRF- $2 \alpha$, mtTFA and AMPK detection was performed after $2,4,6,8$ and $12 \mathrm{~h}$. To assess the effects of the various treatments, the neuron cultures were pretreated with either $1 \mathrm{mM}$ AMPK activator 5-aminoimidazole-4-carboxamide riboside (AICAR) (Sigma-Aldrich) or $50 \mu \mathrm{M}$ resveratrol (Sigma-Aldrich) for $60 \mathrm{~min}$. In order to conduct the inhibitory experiments, the neurons were pretreated with a final concentration of $10 \mu \mathrm{M}$ AMPK antagonist compound C (Sigma-Aldrich) for $30 \mathrm{~min}$, prior to the depolarization experiments.

Reverse transcription-quantitative polymerase chain reaction (RT-qPCR) analysis. Total RNA was extracted from the neurons using TRIzol ${ }^{\circledR}$ (Invitrogen Life Technologies, Carlsbad, CA, USA), and DRR037S (Takara Biotechnology, Co., Ltd, Dalian, China) was used for reverse transcription according to the manufacturer's instructions. The RT-qPCR reaction was performed using a Mastercycler Gradient PCR system (Eppendorf, Hamburg, Germany). The PCR cycling conditions were as follows: Pre-denaturation at $94^{\circ} \mathrm{C}$ for $3 \mathrm{~min}$, followed by 30 cycles of denaturation at $95^{\circ} \mathrm{C}$ for $2 \mathrm{sec}$, annealing at the appropriate temperature (NRF- $2 \alpha, 57^{\circ} \mathrm{C}$; PGC- $1 \alpha, 58^{\circ} \mathrm{C}$; mTFA, $56^{\circ} \mathrm{C}$; $18 \mathrm{~S}, 60^{\circ} \mathrm{C}$ ) for $15 \mathrm{sec}$, extension at $72^{\circ} \mathrm{C}$ for $10 \mathrm{sec}$; and a final extension step at $72^{\circ} \mathrm{C}$ for $7 \mathrm{~min}$.

The following primers were used: PGC- $1 \alpha$ forward, 5'-AGTGTGCTGCTCTGGTTGGTG-3', and reverse, 5'-GGA GGGTCATCGTTTGTGGTC-3'; NRF-2 $\alpha$ forward, 5'-AGG TGACGAGATGGGCTGC-3', and reverse, 5'-CGTTGTCCC CATTTTTGCG-3'; mtTFA forward, 5'-GAAAGCACAAAT CAAGAGGAG-3', and reverse, 5'-CTGCTTTTCATCATG AGACAG-3'; AMPK forward, 5'-ATGCAGCTTCGCAATC GAT-3', and reverse, 5'-GACAGCCAGCCACTCTGGTT-3'; and acetyl-coenzyme A carboxylase (ACC) forward, 5'-AGGAGGGAAGGGAATCAGAA-3', and reverse, 5'-TGTGCTGCAGGAAGATTGAC-3'. The RT-qPCR reaction used $18 \mathrm{~S}$ rRNA to normalize the data (forward, 5'-CGGCTACCACATCCAAGGAA-3', and reverse, 5'-GCTGGAATTACCGCGGCT-3'). The PCR products were then separated by $1 \%$ agarose gel electrophoresis, at $100 \mathrm{~V}$ for $30 \mathrm{~min}$, and the results were analyzed using a Gel Imaging system (Media Cybernetics, Inc., Rockville, MD, USA). The values were normalized to $18 \mathrm{~S}$ rRNA.

AMPK detection. The following conditions were used at various time points prior to $\mathrm{KCl}$ depolarization: $1 \mathrm{~h}$ prior to experimentation, the neurons were treated with a final concentration of $1 \mathrm{mM}$ AMPK activator AICAR or $50 \mu \mathrm{M}$ resveratrol, and $0.5 \mathrm{~h}$ prior to $\mathrm{KCl}$ depolarization, a final concentration of $10 \mu \mathrm{M}$ AMPK inhibitor compound $\mathrm{C}$ was added to the cultured cells, and the AMPK-induced changes in the visual cortical neurons were observed in vitro by RT-qPCR and luminometer.

Measurement of mitochondrial ATP content. The cultured cells were washed with cold PBS ( $\mathrm{pH}$ 7.4), incubated in somatic cell ATP-releasing reagent (Sigma-Aldrich) for $5 \mathrm{~min}$, and harvested using a cell scraper. ATP was used at a 1:100 dilution in order to conduct the ATP detection of working fluid. A total of $100 \mu 1$ ATP detection reagent (Sigma-Aldrich) was added to the harvested cells, and light emission was determined using a BioSpectrometer luminometer (Eppendorf, Hamburg, Germany). The data were compared using an ATP standard curve to calculate the total ATP concentration of each sample.

Statistical analysis. Data are expressed as the mean \pm standard error of the mean from three independent experiments, each repeated in duplicate. Statistically significant differences between the experimental and control groups were deter- 
A
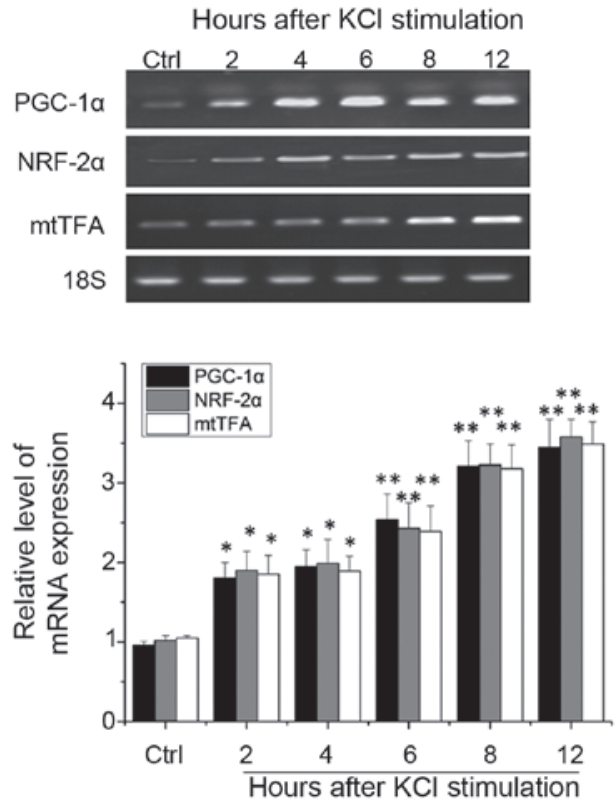

B

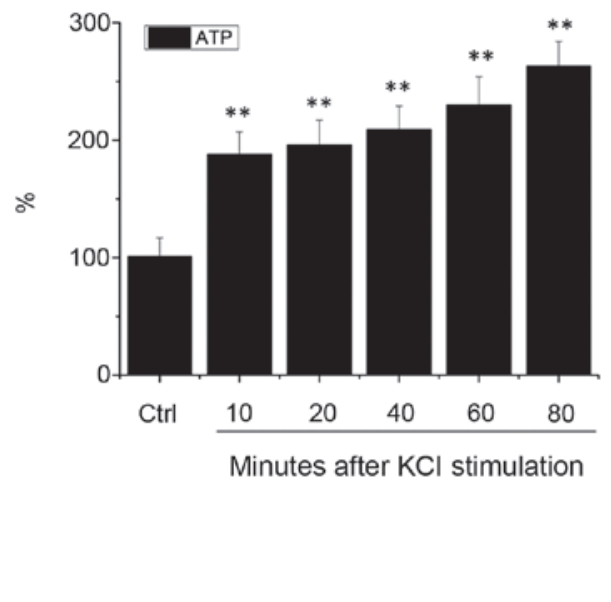

Figure 1. $\mathrm{KCl}$ depolarization upregulates the mRNA expression levels of peroxisome proliferator-activated receptor $\gamma$ coactivator $1 \alpha$ (PGC-1 $\alpha$ ), nuclear respiratory factor- $2 \alpha(\mathrm{NRF}-2 \alpha)$, and mitochondrial transcription factor A (mtTFA) in rat visual cortical neurons. (A) KCl-induced membrane depolarization upregulated the mRNA expression levels of PGC-1 $\alpha$, NRF-2 $\alpha$ and mtTFA. (B) KCl-induced depolarization increased cellular ATP levels in primary visual cortical neurons. ${ }^{*} \mathrm{P}<0.05$ and ${ }^{* *} \mathrm{P}<0.01$, vs. the control neurons.

mined using unpaired Student's t-test, and one-way analysis of variance. $\mathrm{P}<0.05$ was considered to indicate a statistically significant difference.

\section{Results}

$m R N A$ expression levels of $P G C-1 \alpha, N R F-2 \alpha$ and $m t T F A$, and the ATP content in mitochondria. RT-qPCR analysis demonstrated that, as compared with the control group, $2 \mathrm{~h}$ after depolarization, the mRNA expression levels of PGC-1 $\alpha$, NRF-2 $\alpha$ and mtTFA increased significantly in the rat cortical neurons $(\mathrm{P}<0.05)$. After $4 \mathrm{~h}$, the expression levels remained elevated in a time-dependent manner $(\mathrm{P}<0.01)$, with a peak after $12 \mathrm{~h}$ ( $\mathrm{P}<0.01$; Fig. 1A).

These experiments suggest that neuronal excitability of the visual cortex upregulates mitochondrial transcription factors PGC-1 $\alpha$ and NRF- $2 \alpha$ mRNA. To directly observe the association between the neurons in the visual cortex in the excited and metabolic states, the intracellular levels of ATP content in excited visual cortical neurons were measured. Following depolarization for $10 \mathrm{~min}$, the levels of ATP in the cultured neurons increased significantly $(\mathrm{P}<0.01)$. After 20-60 min, the levels of ATP in the cells continued to rise, and after $80 \mathrm{~min}$ of stimulation the elevated ATP levels were maintained, as compared with the control group $(\mathrm{P}<0.01$; Fig. 1B). These results suggest that neuronal excitability in the visual cortex is able to increase the intracellular levels of ATP, and may regulate energy coupling.

mRNA expression levels of AMPK and ACC. Due to the important role of AMPK in neuronal energy metabolism, the present study investigated the mRNA expression levels of AMPK and ACC in the various treatment groups. RT-qPCR analysis revealed that, following $2 \mathrm{~h}$ of depolarization, the
mRNA expression levels of AMPK were significantly elevated $(\mathrm{P}<0.05)$. Similar results were observed in the mRNA expression levels of AMPK kinase downstream component ACC $(\mathrm{P}<0.05)$, suggesting that neurons are able to rapidly activate AMPK protein kinase activity. After 4-8 h, the mRNA expression levels of AMPK and ACC continued to increase $(\mathrm{P}<0.01)$, and after $12 \mathrm{~h}$ this increase in expression was maintained $(\mathrm{P}<0.01)$ (Fig. 2). These results suggest that visual cortical neurons are also able to activate AMPK kinase by excitation.

mRNA expression levels of PGC-1 $\alpha, N R F-2 \alpha$ and $m t T F A$, and ATP content in mitochondria following pretreatment with compound $C$. To investigate whether AMPK is involved in PGC-1 $\alpha$, NRF-2 $\alpha$ and mtTFA excitation-dependent regulation in visual cortical neurons, the neurons were pretreated for 30 min with the AMPK antagonist compound C $(10 \mu \mathrm{M})$, prior to being treated with $25 \mathrm{mM} \mathrm{KCl}$ in order to induce depolarization. RT-qPCR analysis demonstrated that no significant changes in the mRNA expression levels of PGC- $1 \alpha$, NRF- $2 \alpha$ and mtTFA occurred following $\mathrm{KCl}$ treatment for 2, 4, 6, 8 and $12 \mathrm{~h}$, as compared with the control group ( $\mathrm{P}>0.05$; Fig. $3 \mathrm{~A}$ ). These results suggest that the AMPK antagonist compound $\mathrm{C}$ inhibited the depolarization-induced upregulation of PGC-1 $\alpha$, NRF- $2 \alpha$, and mtTFA mRNA. Furthermore, no significant changes in the mRNA expression levels of AMPK and ACC were observed $(\mathrm{P}>0.05$; Fig. $3 \mathrm{~B})$. These results suggest that in visual cortical neurons, the upregulation of PGC-1 $\alpha$, NRF- $2 \alpha$ and mtTFA are regulated by AMPK-induced excitation.

The measurement of the levels of ATP after $5 \mathrm{~h}$ of $\mathrm{KCl}$ treatment revealed that the intracellular ATP levels in visual cortical neurons are significantly upregulated, as compared with the control group $(\mathrm{P}<0.01)$. Furthermore, pretreatment with AMPK antagonist compound $\mathrm{C}$ was able to completely block the upregulation of ATP in rat visual cortical neurons 

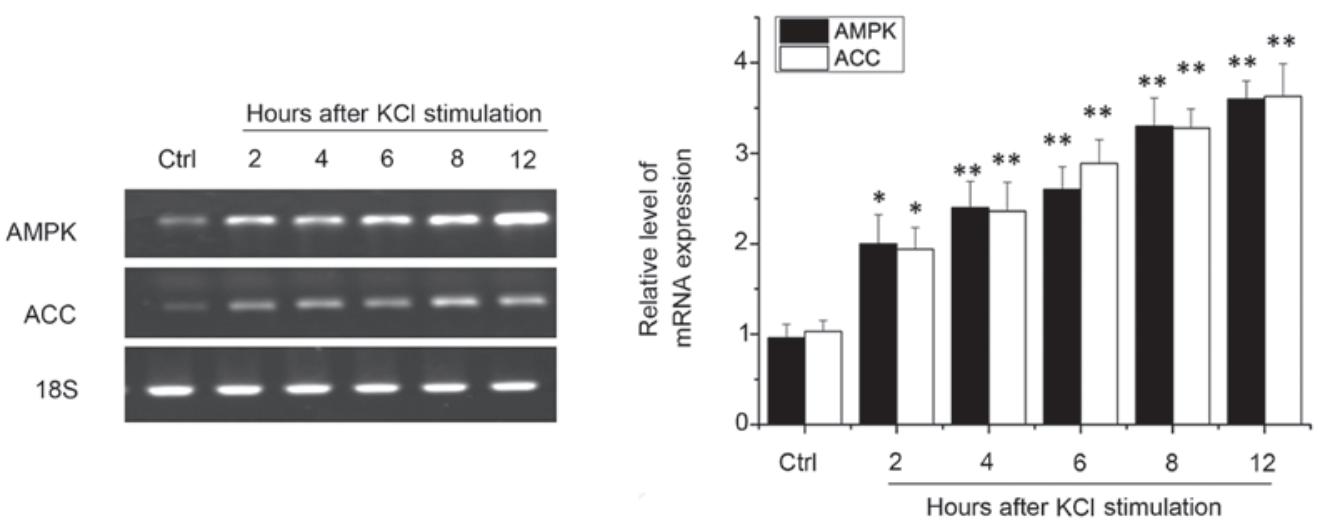

Figure 2. $\mathrm{KCl}$ depolarization upregulates adenosine monophosphate-activated protein kinase (AMPK) and acetyl-coenzyme A carboxylase (ACC) mRNA expression. " $\mathrm{P}<0.05$ and ${ }^{* *} \mathrm{P}<0.01$, vs. the control neurons.

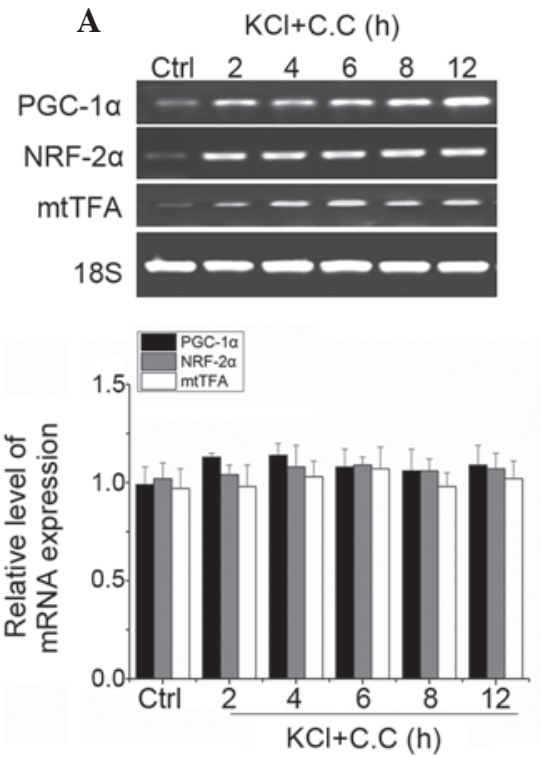

B

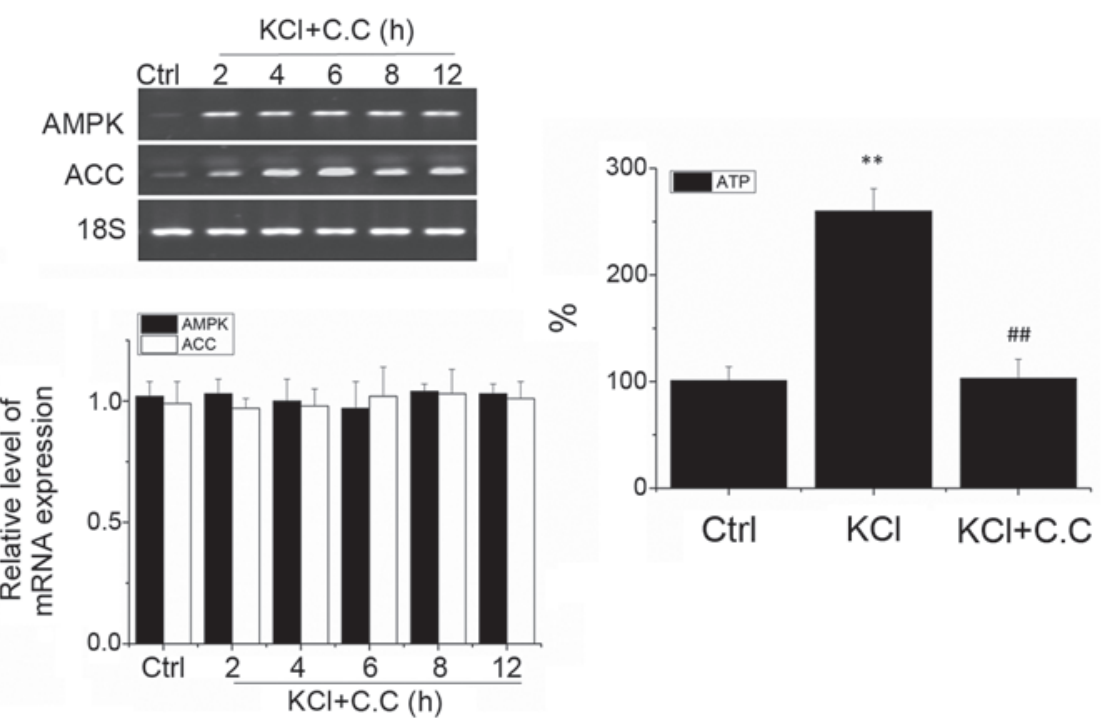

Figure 3. Inhibition of adenosine monophosphate-activated protein kinase (AMPK) by compound C (CC) suppresses the KCl-induced mRNA expression levels of peroxisome proliferator-activated receptor $\gamma$ coactivator $1 \alpha$ (PGC-1 $\alpha$ ), nuclear respiratory factor- $\alpha$ (NRF-2 $\alpha$ ) and mitochondrial transcription factor A (mtTFA). (A) Compound C suppressed KCl-induced PGC-1 $\alpha$, NRF-2 $\alpha$ and mtTFA expression. (B) Compound C suppressed KCl-induced AMPK and acetyl-coenzyme A carboxylase (ACC) expression. (C) KCl-induced ATP production was inhibited by compound C. ${ }^{* *} \mathrm{P}<0.01$, vs. the control neurons and ${ }^{\# \#} \mathrm{P}<0.01$, vs. the KCl-treated neurons.

( $\mathrm{P}>0.05$ as compared with the control group, and $\mathrm{P}<0.01$ as compared with the $\mathrm{KCl}$ group; Fig. 3C). These results suggest that AMPK is an important regulator of energy excitation coupling in visual cortical neurons.

mRNA expression levels of PGC-1 $\alpha, N R F-2 \alpha$ and mtTFA, and the levels of ATP mitochondria treated with AICAR and resveratrol. The results of the present study demonstrated that AMPK is an important regulator of visual cortical neuron excitation energy coupling, and may mediate visual cortical neuron NRF- $2 \alpha$ excitation-dependent regulation. To further investigate the association between AMPK and NRF- $2 \alpha$, the effects of the AMPK agonist AICAR and resveratrol on the expression levels of NRF- $2 \alpha$ in visual cortical neurons were observed. As compared with the control group, treatment with $1 \mathrm{mM}$ AICAR for $2 \mathrm{~h}$ (Fig. 4A) or $20 \mu \mathrm{M}$ resveratrol for $2 \mathrm{~h}$ (Fig. 4B) significantly increased the
mRNA expression levels of AMPK, ACC and NRF-2 $\alpha$ in the neurons $(\mathrm{P}<0.01)$. These results suggest that the activity levels of AMPK are regulated by AICAR and resveratrol. Furthermore, the neuronal excitation induced by resveratrol was inhibited by the AMPK antagonist compound C $(10 \mu \mathrm{M})$, and the activation of NRF-2 $\alpha$ mRNA expression was also inhibited (Fig. 4C). These results suggest that the regulatory effects of resveratrol on the activation of NRF- $2 \alpha$ in neurons is dependent on AMPK activation. Therefore, activation of AMPK is able to effectively regulate the NRF- $2 \alpha$ expression in cortical neurons.

To investigate the effects of AMPK on visual cortical neurons, the present study examined neuronal ATP levels. Treatment with $1 \mathrm{mM}$ AICAR and $20 \mu \mathrm{M}$ resveratrol significantly increased neuronal ATP levels $(\mathrm{P}<0.01)$. Furthermore, pretreatment with the AMPK antagonist compound $\mathrm{C}$ was able to inhibit resveratrol-induced upregulation of ATP 
A
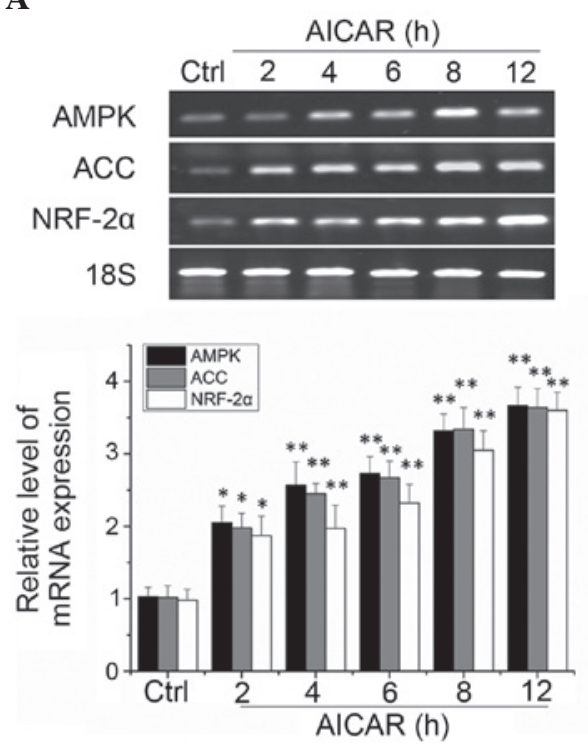

C
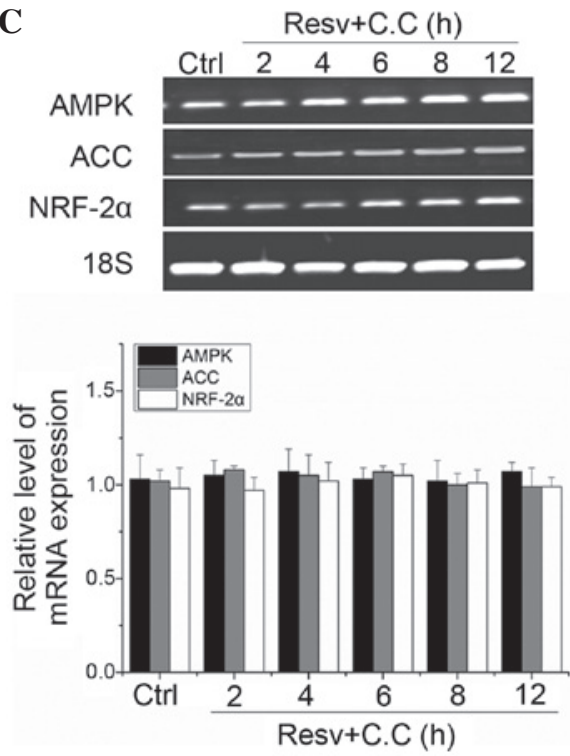

B
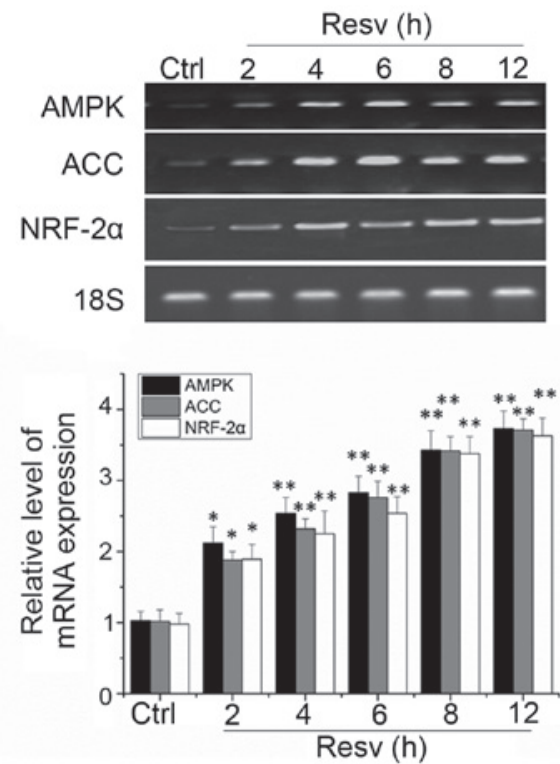

D

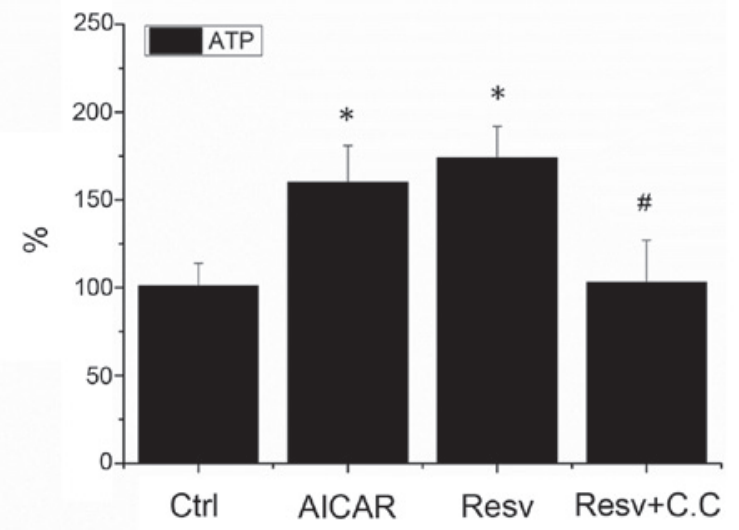

Figure 4. 5-Aminoimidazole-4-carboxamide riboside (AICAR) and resveratrol upregulate adenosine monophosphate-activated protein kinase (AMPK), acetyl-coenzyme A carboxylase (ACC) and nuclear respiratory factor-2 $\alpha$ (NRF-2 $\alpha$ ) mRNA expression in rat visual cortical neurons. (A) Treatment with AICAR increased the mRNA expression levels of AMPK, ACC and NRF-2 $\alpha$. (B) Treatment with resveratrol (Resv) increased the mRNA expression levels of AMPK, ACC and NRF-2 $\alpha$. (C) AMPK inhibitor compound C suppressed resveratrol-induced AMPK, ACC and NRF-2 $\alpha$ expression. (D) Cellular ATP levels were increased following treatment with AICAR and resveratrol, and resveratrol-induced ATP levels were inhibited by compound $\mathrm{C}$. ${ }^{*} \mathrm{P}<0.05$, vs. the control neurons and ${ }^{\#} \mathrm{P}<0.05$, vs. the resveratrol-treated neurons.

compared ( $\mathrm{P}>0.005$, as compared with the control group, and $\mathrm{P}<0.01$, as compared with the resveratrol group; Fig. 4D). These results indicate that activation of AMPK is able to adjust the energy levels in visual cortical neurons.

\section{Discussion}

Energy metabolism is an important source of life, and the central nervous system consumes a large amount of energy. The brain accounts for only $2 \%$ of the total body weight, but consumes $20 \%$ of its total glucose (13). Little energy is stored within the central nervous system, and a balance between energy supply and consumption is maintained. The appropriate energy balance regulation of such a high energy consuming and low energy storage system is essential (14).
The role of visual cortical neurons is largely dependent on the energy provided by the ATP produced by the oxidation of glucose. The high energy demand of the central nervous system derives from two processes: The basic process of nurturing (such as the maintenance of cellular membrane integrity and protein synthesis), and physiological function (such as the electrical activity of neurons). The majority of the visual cortical neuron energy expenditure is utilized to maintain visual stimulation.

Plasticity of the neuronal energy metabolism and neuronal excitability are tightly coupled processes. Neurons are highly dependent on OXPHOS to produce ATP (15). For visual cortical neurons, the neuronal excitation energy coupling mechanism is particularly important. Due to the fact that visual cortex plasticity depends on visual experience, the role of normal 
visual stimulus-induced neuronal excitability in maintaining neuronal physiological function in the visual cortex is of great significance (16). Research conducted in the primate animal model, and clinical observations of amblyopia demonstrated that mitochondrial biogenesis and dysfunction are important factors in optic atrophy, amblyopia, and other visual pathway diseases. These results suggested that mitochondrial dysfunction is an important factor in visual deprivation and visual functional changes in cortical neurons, and the maintenance the mitochondrial energy balance may be important for the correct functioning of cortical neurons (17).

Visual stimulation and experience are important factors in the development of visual cortical neurons. The composition, function, and synaptic transmission and distribution of neurons in the visual cortex vary according to visual environment and experience, a process termed experience-dependent plasticity of visual development (18). Inhibition of the input of visual information negatively affects the development of the visual cortex. In addition, energy metabolism may regulate visual cortical neuron activity levels (19), with altered gene expression resulting in changes in neuronal plasticity. These neuronal plasticity changes, including mitochondrial dysfunction, in visual cortical neurons are a central mechanism underlying visual deprivation-induced amblyopia (20).

OXPHOS takes place in the mitochondria (21). The process of OXPHOS is highly dependent on NRFs (22). The NRF family includes NRF-1 and NRF-2. The main function of NRFs is to regulate the expression of respiratory chain and mitochondrial RNA endonuclease and mtTFA. NRF-2 consists of two subunits, NRF-2 $\alpha$ and NRF- $2 \beta$. The primary function of NRF-2 $\alpha$ is the regulation of genes associated with the mitochondrial energy metabolism. The functional binding sites of NRF- $2 \alpha$ include a COX subunit promoter, ATP synthase $\beta$, mtTFA, and mtTFB (23). NRF-2 $\alpha$ regulates the activity levels of the COX gene in the brain, when the energy demand of the cells change (6). Notably, NRF-2 $\alpha$ has a role in the regulation of cellular respiratory function (24).

mtTFA and mtTFB, encoded by nDNA, are transcription factors that regulate the transcription and replication of mtDNA (25). mtTFA regulates transcriptional activity, which also depends on the synergistic effects of NRF-2 $\alpha$. COX is the rate limiting enzyme in the production of ATP, and is the only molecule able to transfer electrons to cytochrome $c$ (26). The formation of COX is dependent on the coordinated function of mtDNA and nDNA. NRF-2 $\alpha$ is involved in this important and complex process. The expression of COX is directly regulated by NRF- $2 \alpha$. In addition, NRF- $2 \alpha$ is able to activate mtTFA and mtTFB in an indirect manner, in order to regulate transcription.

PGC-1 $\alpha$ is an important coregulator of mitochondrial hyperplasia. One of the primary functions of PGC-1 $\alpha$ is to promote the transcription of NRF-1 and NRF-2, and to coordinate the function of the mitochondrial respiratory chain in order to meet the requirements of the various cellular energy states (27). These effects may be further promoted by the expression of OXPHOS components, such as mtTFA, mtTFB, and COX II and IV (11).

The results of the present study demonstrated that in response to $\mathrm{KCl}$ depolarization, visual cortical neurons upregulate PGC-1 $\alpha$, NRF-2 $\alpha$ and mtTFA expression. This in turn enhanced the ability of mitochondria to produce ATP. Additionally, the results suggested that the PGC- $1 \alpha, \mathrm{NRF}-2 \alpha$ and mtTFA signaling pathways are associated with neuronal activity in rat visual cortical neurons.

Compared with a previous study (12), the expression levels of NRF- $2 \alpha$ increased later than those of NRF-1. This may suggest that in the visual cortex, NRFs have a different pattern of response.

AMPK is a widely distributed serine/threonine protein kinase in eukaryotic cells. It is activated when cellular energy is insufficient, promotes the oxidation of fatty acids and glucose transport, and increases intracellular ATP levels. In addition, AMPK inhibits the synthesis of glycogen, fat, and cholesterol and reduces the consumption of ATP (28).

AMPK has a multi-level role in neuronal energy metabolism. Changes in the activity levels of AMPK in hypothalamic neurons may regulate individual appetite and feeding behavior, in order to control the energy balance via intake and consumption. An AMPK $\alpha 2$ knockout mouse model demonstrated that sympathetic nervous system AMPK is involved in the regulation of insulin sensitivity in peripheral tissues (29). Activation of AMPK in the brain protects against neuronal injury induced by energy deprivation (30) and AMPK mutations may cause a broad spectrum of neurodegenerative changes at the cellular level (31). AMPK regulation is sensitive to the ratio of intracellular adenosine mono-phosphate and ATP; when the intracellular levels of ATP decrease, AMPK rapidly activates the regulation of the intracellular energy metabolism. Changes in intracellular $\mathrm{Ca}^{2+}$ concentration may also activate AMPK. AMPK is an important factor in the regulation of the energy balance of the nervous system. A previous study demonstrated that AMPK is able to activate NRFs and change the levels of excitation in mitochondrial synthesis (32).

$\mathrm{KCl}$-induced membrane depolarization significantly stimulated neuronal AMPK, and the expression of its downstream kinase, ACC. These results suggest that AMPK is activated in neurons by neuronal activity. Furthermore, the depolarization of visual cortical neurons resulted in significant increases in neuronal ATP levels. The association between neuronal excitability and energy response was inhibited by the AMPK inhibitor compound C. These results suggested that AMPK has an important role in visual cortical neuron energy coupling. Using the AMPK agonist AICAR to activate AMPK resulted in significant upregulation of PGC-1 $\alpha$ and NRF-2 $\alpha$ expression in visual cortical neurons. In addition, previous studies have demonstrated that resveratrol is an effective activator of AMPK (33-35). Similarly, the results of the present study indicated that resveratrol is able to activate AMPK kinase activity in visual cortical neurons, and the upregulation of neuronal PGC-1 $\alpha$ by resveratrol suggests a dependence on NRF-2 $\alpha$ expression levels. These effects were suppressed by the AMPK inhibitor compound C. These results demonstrate that resveratrol significantly increased the expression levels of PGC- $1 \alpha$ and NRF- $2 \alpha$ in visual cortical neurons via the AMPK signaling pathway. The activation of AMPK led to the upregulation of PGC-1 $\alpha$ and NRF-2 $\alpha$ expression, and eventually to a neuronal energy response and a significant increase in the intracellular levels of ATP.

In conclusion, the results of the present study demonstrate that AMPK participates in the energy metabolism of visual 
cortical neurons. In addition, AMPK is an important transcriptional regulator of the neuronal energy excitation response, which is mediated by the PGC- $1 \alpha$ and NRF-2 $\alpha$ signaling pathway.

\section{Acknowledgements}

No financial support was received for the present study.

\section{References}

1. Kann $\mathrm{O}$ and Kovács R: Mitochondria and neuronal activity. Am J Physiol Cell Physiol 292: C641-C657, 2007.

2. Leruez S, Amati-Bonneau P, Verny C, Reynier P, Procaccio V, Bonneau D and Milea D: Mitochondrial dysfunction affecting visual pathways. Rev Neurol (Paris) 170: 344-354, 2014.

3. Nisoli E, Clementi E, Moncada S and Carruba MO: Mitochondrial biogenesis as a cellular signaling framework. Biochem Pharmacol 67: 1-15, 2004.

4. Kuo JJ, Chang HH, Tsai TH and Lee TY: Curcumin ameliorates mitochondrial dysfunction associated with inhibition of gluconeogenesis in free fatty acid-mediated hepatic lipoapoptosis. Int J Mol Med 30: 643-649, 2012.

5. Shi Y, Dierckx A, Wanrooij PH, Wanrooij S, Larsson NG, Wilhelmsson LM, Falkenberg $\mathrm{M}$ and Gustafsson $\mathrm{CM}$ : Mammalian transcription factor $\mathrm{A}$ is a core component of the mitochondrial transcription machinery. Proc Natl Acad Sci USA 109: 16510-16515, 2012

6. Ongwijitwat S, Liang HL, Graboyes EM and Wong-Riley MT: Nuclear respiratory factor 2 senses changing cellular energy demands and its silencing down-regulates cytochrome oxidase and other target gene mRNAs. Gene 374: 39-49, 2006.

7. Li WY, Yao CX, Zhang SF, Wang SL, Wang TQ, Xiong CJ, Li YB and Zang MX: Improvement of myocardial lipid accumulation and prevention of PGC-1alpha induction by fenofibrate. Mol Med Rep 5: 1396-1400, 2012.

8. Hossain MB, Ji P, Anish R, Jacobson RH and Takada S Poly(ADP-ribose) polymerase 1 interacts with nuclear respiratory factor 1 (NRF-1) and plays a role in NRF-1 transcriptional regulation. J Biol Chem 284: 8621-8632, 2009.

9. Manwani B and McCullough LD: Function of the master energy regulator adenosine monophosphate-activated protein kinase in stroke. J Neurosci Res 91: 1018-1029, 2013.

10. Rousset CI, Leiper FC, Kichev A, Gressens P, Carling D, Hagberg $\mathrm{H}$ and Thornton $\mathrm{C}$ : A dual role for AMP-activated protein kinase (AMPK) during neonatal hypoxic-ischaemic brain injury in mice. J Neurochem 133: 242-252, 2015.

11. Scarpulla RC: Metabolic control of mitochondrial biogenesis through the PGC-1 family regulatory network. Biochim Biophys Acta 1813: 1269-1278, 2011.

12. Yang SJ, Liang HL and Wong-Riley MT: Activity-dependent transcriptional regulation of nuclear respiratory factor-1 in cultured rat visual cortical neurons. Neuroscience 141: 1181-1192, 2006.

13. Zhao Q, Wang S, Li Y, Wang P, Li S, Guo Y and Yao R: The role of the mitochondrial calcium uniporter in cerebral ischemia/reperfusion injury in rats involves regulation of mitochondrial energy metabolism. Mol Med Rep 7: 1073-1080, 2013.

14. Rodriguez-Rodriguez P, Almeida A and Bolaños JP: Brain energy metabolism in glutamate-receptor activation and excitotoxicity: role for APC/C-Cdh1 in the balance glycolysis/pentose phosphate pathway. Neurochem Int 62: 750-756, 2013

15. Breuer ME, Koopman WJ, Koene S, Nooteboom M, Rodenburg RJ, Willems PH and Smeitink JA: The role of mitochondrial OXPHOS dysfunction in the development of neurologic diseases. Neurobiol Dis 51: 27-34, 2013.

16. Patterson CA, Duijnhouwer J, Wissig SC, Krekelberg B and Kohn A: Similar adaptation effects in primary visual cortex and area MT of the macaque monkey under matched stimulus conditions. J Neurophysiol 111: 1203-1213, 2014.
17. Yu Wai Man CY, Chinnery PF and Griffiths PG: Optic neuropathies - importance of spatial distribution of mitochondria as well as function. Med Hypotheses 65: 1038-1042, 2005.

18. Sur M, Nagakura I, Chen N and Sugihara H: Mechanisms of plasticity in the developing and adult visual cortex. Prog Brain Res 207: 243-254, 2013

19. Chevrollier A, Guillet V, Loiseau D, Gueguen N, de Crescenzo MA, Verny C, Ferre M, Dollfus H, Odent S, Milea D, et al: Hereditary optic neuropathies share a common mitochondrial coupling defect. Ann Neurol 63: 794-798, 2008.

20. Nie F and Wong-Riley M: Nuclear respiratory factor-2 subunit protein: Correlation with cytochrome oxidase and regulation by functional activity in the monkey primary visual cortex. J Comp Neurol 404: 310-320, 1999.

21. Tao M, You CP, Zhao RR, Liu SJ, Zhang ZH, Zhang C and Liu Y: Animal mitochondria: Evolution, function, and disease. Curr Mol Med 14: 115-124, 2014.

22. Wong-Riley MT: Bigenomic regulation of cytochrome c oxidase in neurons and the tight coupling between neuronal activity and energy metabolism. Adv Exp Med Biol 748: 283-304, 2012.

23. Escrivá H, Rodríguez-Peña A and Vallejo CG: Expression of mitochondrial genes and of the transcription factors involved in the biogenesis of mitochondria Tfam, NRF-1 and NRF-2, in rat liver, testis and brain. Biochimie 81: 965-971, 1999.

24. D'Souza D, Lai RY, Suen M and Hood DA: mRNA stability as a function of striated muscle oxidative capacity. Am J Physiol Regul Integr Comp Physiol 303: R408-R417, 2012.

25. Girodet PO, Ozier A, Bara I, Tunon de Lara JM, Marthan R and Berger P: Airway remodeling in asthma: New mechanisms and potential for pharmacological intervention. Pharmacol Ther 130: 325-337, 2011.

26. Mayall TP, Bjarnason I, Khoo UY, Peters TJ and Macpherson AJ: Mitochondrial gene expression in small intestinal epithelial cells. Biochem J 308: 665-671, 1995.

27. Venditti P, Bari A, Di Stefano L, Cardone A, Della Ragione F, D'Esposito $\mathrm{M}$ and Di Meo S: Involvement of PGC-1, NRF-1, and NRF-2 in metabolic response by rat liver to hormonal and environmental signals. Mol Cell Endocrinol 305: 22-29, 2009.

28. Williams T, Courchet J, Viollet B, Brenman JE and Polleux F: AMP-activated protein kinase (AMPK) activity is not required for neuronal development but regulates axogenesis during metabolic stress. Proc Natl Acad Sci USA 108: 5849-5854, 2011.

29. Wang P, Zhang RY, Song J, Guan YF, Xu TY, Du H, Viollet B and Miao CY: Loss of AMP-activated protein kinase- $\alpha 2$ impairs the insulin-sensitizing effect of calorie restriction in skeletal muscle. Diabetes 61: 1051-1061, 2012.

30. Klip A, Schertzer JD, Bilan PJ, Thong F and Antonescu C: Regulation of glucose transporter 4 traffic by energy deprivation from mitochondrial compromise. Acta Physiol (Oxf) 196: 27-35, 2009.

31. Garcia-Roves PM, Osler ME, Holmström MH and Zierath JR: Gain-of-function R225Q mutation in AMP-activated protein kinase gamma3 subunit increases mitochondrial biogenesis in glycolytic skeletal muscle. J Biol Chem 283: 35724-35734, 2008

32. Yan W, Zhang H, Liu P, Wang H, Liu J, Gao C, Liu Y, Lian K, Yang L, Sun L, et al: Impaired mitochondrial biogenesis due to dysfunctional adiponectin-AMPK-PGC-1 $\alpha$ signaling contributing to increased vulnerability in diabetic heart. Basic Res Cardiol 108: 329, 2013.

33. Choi YJ, Suh HR, Yoon Y, Lee KJ, Kim DG, Kim S and Lee BH: Protective effect of resveratrol derivatives on high-fat diet induced fatty liver by activating AMP-activated protein kinase. Arch Pharm Res 37: 1169-1176, 2014.

34. Shang J, Chen LL, Xiao FX, Sun H, Ding HC and Xiao H: Resveratrol improves non-alcoholic fatty liver disease by activating AMP-activated protein kinase. Acta Pharmacol Sin 29: 698-706, 2008

35. Vingtdeux V, Giliberto L, Zhao H, Chandakkar P, Wu Q, Simon JE, Janle EM, Lobo J, Ferruzzi MG, Davies P, and Marambaud P: AMP-activated protein kinase signaling activation by resveratrol modulates amyloid-beta peptide metabolism. J Biol Chem 285: 9100-9113, 2010 